INTERNATIONAL JOURNAL OF RESEARCHES IN BIOSCIENCES, AGRICULTURE \& TECHNOLOGY (c) VISHWASHANTI MULTIPURPOSE SOCIETY (Global Peace Multipurpose Society) R. No. MH-659/13(N) www.vmsindia.org

\title{
Bianchi Type-I Perfect Fluid Model in Bimetric Theory of Gravitation
}

\author{
Vijay Lepse \\ Science College, Pauni-441910, India \\ vijaylepse@gmail.com
}

\begin{abstract}
Bianchi type-I cosmological model as related to perfect fluidinbimetric theory of gravitation have been deduced. The perfect fluid model has hyperbolic geometry and all its physical parameters are also hyperbolic in nature and therefore they have been studied from hyperbolic geometric view point. All these models are isotropize and shear-less. Other geometrical and physical behaviors of the models have also been studied.
\end{abstract}

Keywords: Bianchi type-I; bimetric gravitational theory; cosmology; hyperbolic geometric; isotropize; geometry

\section{Introduction}

Rosen's $(1973,1975)$ bimetric theory of gravitation is one of the altematives to general relativity and it is free from singularities appearing in the big-bang of cosmological models and it obeys the principle of covariance and equivalence of the general relativity. Therefore, the people are interested in investigating the cosmological models of the universe in bimetric theory of gravitation based on two matrices; one is Riemannian metric which described the geometry of curved space time, and the second is flat metric which refers to the geometry of the empty universe (no matter but gravitation is there) and described the initial forces.

The Rosen's field equations in bimetric theory of gravitation are

$$
N^{j}-\frac{1}{2} N \delta=-T^{j}
$$

where

$$
N^{\prime}=\frac{1}{2} \cdot\left(g^{\prime} g\right) \text {. }
$$

$N=g^{3} N$, is the Rosen scalar. The vertical bar $(\mid)$ stands for $\gamma$-covariant differentiation where $g=\operatorname{det}\left(g_{j}\right)$ and $\gamma=\operatorname{det}\left(\gamma_{b}\right)$. Many researchers have developed the theory and investigated many cosmological models of the universe in bimetric theory of gravitation and in general relativity, and studied their behavior geometrically and physically [Karade (1980), Israelit (1981), Reddy et al. (1989, 1998), Mohanty et al. (2002), Bali (2003a, 2003b, 2005, 2006, 2007), Katore (2006), Khadekar (2007), Borkar (2010a, 2013, 2014a, 2014b), Gaikwad (2011)]. Athough the non-existence of Bianchi types I, III, V and V/locosmological models 
with perfect fluid in the Rosen's bimetric theory of gravitation have been shown by Reddy et al. (1989, 1998), Mohanty et al. (2002) and Borkar et al. (2010b) have deduced the existence of Bianchi type I magnetized cosmological model in Bimetric theory of gravitation and studied its geometrical and physical properties.

\section{Solutions of Rosen's fieldequations}

We consider the Bianchi Type I metric [Borkar et al. (2010b)] in the form

$$
d s^{2}=-\mathbf{d t ^ { 2 }}+\mathbf{A}^{2} \mathbf{d} \mathbf{x}^{2}+\mathbf{B}^{2} \mathbf{d} \mathbf{y}^{2}+\mathbf{C}^{2} \mathbf{d z ^ { 2 }},
$$

where $A, B$ and $C$ are functions of $t$ only.

The flat metric corresponding to metric (2) is

$$
d \eta^{2}=-d t^{2}+d x^{2}+d y^{2}+d z^{2}
$$

The energy momentum tensor $T^{j}$ of the source (Sharif et al. (2013)) is given by

$$
T^{\prime}=(\rho+p) v v^{\prime}+p \delta, \quad,
$$

with

$$
y v^{\prime}=-1
$$

Here, pand paretheenergydensity andpressureofperfectfluid, respectivelyand $v$ is the flow vector. The quantity $\theta$ s the scalar of expansion which is given by

$$
\theta=v^{i}
$$

We assume the coordinates to be co-moving, so that

$$
v^{1}=v^{2}=v^{3}=0, \quad v^{4}=-1
$$

Equation (4) of energy momentum tensor yield

$$
T_{1}^{4}=T_{2}^{2}=T_{3}^{3}=\underset{3}{p} \quad \text { and } \quad T_{4}^{4}=-\rho
$$

The pressure $p$ and the density pare related by an equation of state $\quad p=\gamma \rho, \quad 0 \leq \gamma \leq 1$. The Rosen's field Equations (1) for the metric (2) and (3) with the help of (7) give the differential equations 


$$
\begin{aligned}
& -\frac{\sqrt{A}}{A}+\frac{\bar{B}}{B}+\frac{\bar{C}}{C}+\frac{A^{2}}{A^{2}}-\frac{B^{2}}{B^{2}}-\frac{C^{2}}{C^{2}}=-16 \pi A B C p, \\
& \frac{A}{A}-\frac{B}{B}+\frac{E}{C}-\frac{A^{2}}{A^{2}}+\frac{B^{2}}{m}-\frac{\bar{C}^{0}}{-\infty}=-16 \pi A B C p,
\end{aligned}
$$

From Equations (8) - (10), we write

$$
A=B \quad=C \text {. }
$$

Also, from Equations (8) and (11), we get

$$
\frac{A}{A^{2}}-\frac{A}{A}=-8 \pi A B C(p+\rho) .
$$

The volume $V$ is a function of $t$ and it is

$$
V=A B C \text {. }
$$

From Equation (12), we write

$$
V=A^{3}, \quad \text { i.e., } \quad A=B=C=\quad V^{\frac{1}{3}} \text {. }
$$

From Equations (13) - (15), we get

$$
\frac{\bar{V}}{V} \quad \frac{\bar{V}^{2}}{V^{2}}=24 \pi V(p+\rho) \text {. }
$$

\section{The perfect fluid model with physicalsignificance}

The conservation law for the energy-momentum tensor from Equation (4), we write

$$
\rho=-\frac{\bar{v}}{V}(p+\rho)
$$

The perfect fluid obeys the equation of state

$$
p_{p p}=\gamma \quad \rho_{\rho F}, \quad 0 \leq \gamma \leq 1
$$

Here, $\gamma=0$ (Dust Universe), $\gamma=1 / 3$ (Radiation Universe), $\gamma={ }^{(1} \quad Y_{\text {(HardUniverse) }}$

and $\gamma=1$ (Zel'dovich Universe or stiff matter).

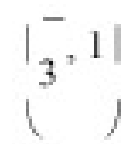

In a co-moving coordinate, the conservation law of energy momentum tensor (17), for the perfect fluid and dark energy is

$$
\hat{p}_{C E}+\quad \hat{\rho}_{P F}=-\hat{V}_{V}\left(\rho, \rho_{D F}+p_{C E}+p_{P F}\right) \text {, }
$$


from which we have

$$
\left.\rho_{D E S}+\frac{V}{V}(\rho)+p_{D E}\right)=0,
$$

and

$$
\bar{p}_{T F}+\frac{\bar{V}_{V}}{V}\left(\rho_{F P}+p_{P F}\right)=0 .
$$

From Equations (18) and (21), we write

$$
\rho_{P F}=\frac{c_{1}}{V^{(1+\eta)}}, \quad p_{P F}=\frac{j c_{1}}{(1+\eta)},
$$

where $c_{1}$ is an integration constant. Using

Equation (22), Equation (16)infers

$$
\bar{V}= \pm \sqrt{c_{2} V^{2}-48 \pi \frac{(1+\gamma)}{\gamma} c_{1} V^{2-\gamma}},
$$

where $c_{2}$ is a constant of integration.

This Equation (23) has a solution

$$
\int \frac{d V}{\sqrt{c_{2} V^{2}-48 \frac{\pi}{\pi}\left(1+\frac{1}{\gamma}\right) / V_{1}^{2-\gamma}}}=t+t_{0}
$$

where $t_{0}$ is constant of integration and is taken to be zero. So

$$
\int \frac{d V}{\sqrt{c_{2} V^{2}-48 \pi}\left(1+\frac{1}{\gamma}\right) V_{1}^{2-\gamma}}=t
$$

We study the model in view of the values of $\gamma$.

Case(i) For $\gamma=0$, the integral Equation (25) has a singularity in dust regime. The model does not predict or does not allow a phase in which the universe is dominated by dust.

Case (ii) For $\gamma=1 / 3$, the solution of integral Equation (25) is

$$
V=\left[c_{3} / \sqrt{c_{2}} \cosh \left(\sqrt{c_{2}} / 6\right) r\right]^{b}
$$


where $c_{2}$ and $c_{3}$ are positive constants. For properchoice $\quad\left(c_{3} / \sqrt{c_{2}}\right)=1$ and $\left(\sqrt{c_{2}} / 6\right)=\alpha$ (constant), we have

$$
V=(\cosh \alpha t)^{6},
$$

and the scale factors $A, B$ and $C$, (from Equation (15)) have the values

$$
A=B=C=(\cosh (\alpha t))^{2} .
$$

The line element (2) becomes

$$
d s^{2}=-d t^{2}+(\cosh (\alpha t))^{4}\left(d x^{2}+d y^{2}+d z^{2}\right) .
$$

This is Bianchi type I perfect fluid cosmological model in the absence of dark energy in Bimetric theory of gravitation.

It is to be noted that this model has hyperbolic geometry. At $t=0, V=A=B=C=1$ and they arehyperbolicallyincreasinginnaturewithincreasingtimerandattaininfinitevalues, as $r \rightarrow \infty$. Thisshowsthatthemodelstartswithnonzerovolumeandthevolumeandscale factors are hyperbolically increasing with increase in time $t$ and they go over to infinity at a later stage.

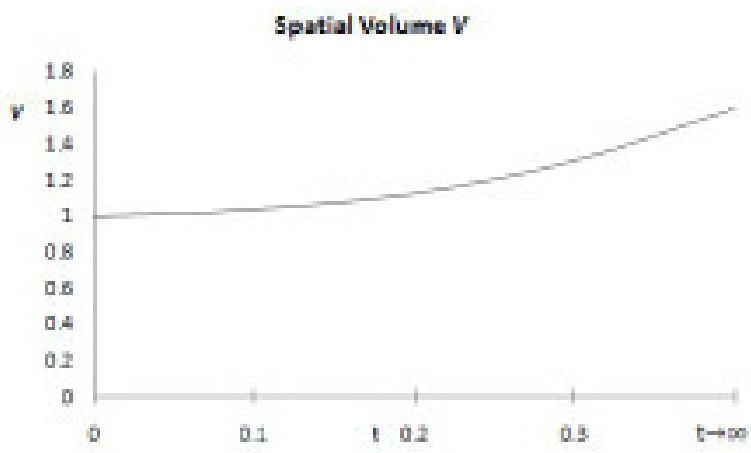

Figure 1. $V$ vst

This hyperbolic geometrical view point of the model will definitely be a benefit to the mathematical and physical community and the people of observational data to search such type of geometry. Recently, Ungar (2009) observed the hyperbolic geometry view point of Einstein's special relativity.

The energy density $\rho_{P F}$ and the isotropic pressure $p_{P F}$ of the perfect fluid model are

$$
\rho_{P F}=3 p_{P F} \quad c_{/} /(\cosh (\alpha t))^{4 / 3} .
$$

The physical quantities like scalar expansion $\theta$,anisotropic parameter $A$, the shear scalar $\sigma$ and the deceleration parameter $q$ have been calculated as 
$\theta=6 \alpha \tanh (\alpha t)$

$A=\sigma=0$,

$q=-\left(1 / 2(\sinh (\alpha t))^{2}\right)-1$

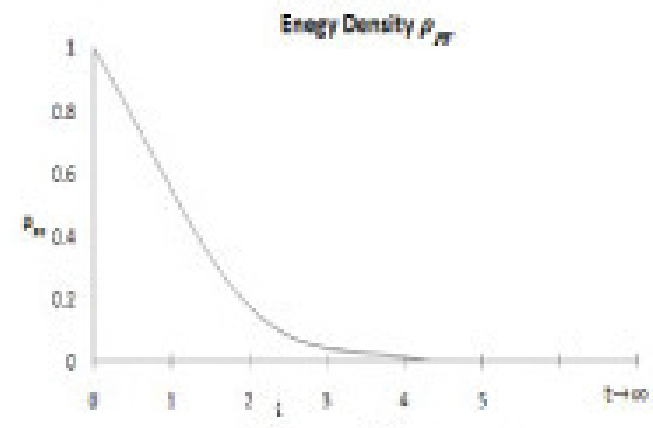

Figure 2. $\rho_{P F}$ vst

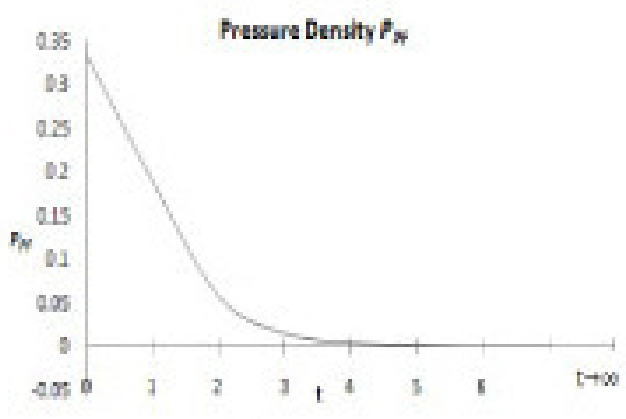

Figure 3. $p_{P Q} \mathrm{VSt}$

Figure 2 and Figure 3 show the nature of density and pressure (in similar nature) of the perfect fluid model. At $t=0$, pressure and density attain the maximum value and theyare decreasing very fast as $t$ is increasing, and approaches zero value for 4.5 (appro.) $<t<\infty$. Thisshowsthatthisradiatinguniversehasveryhighdensityandpressureinthebeginning and its density and pressure go on decreasing and attain zero value for $t \geq 4.5$ i.e, this radiating universe has the matter for some interval of time $0 \leq t \leq 4.5$ (appro.) and the model admit the vacuum case forever for $t \geq 4.5$.

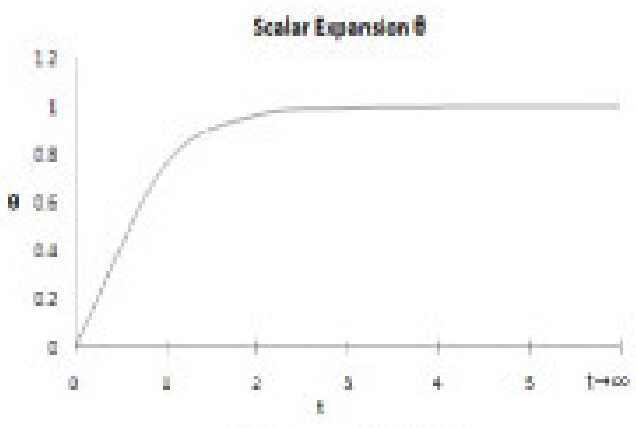

Figure 4. Avst

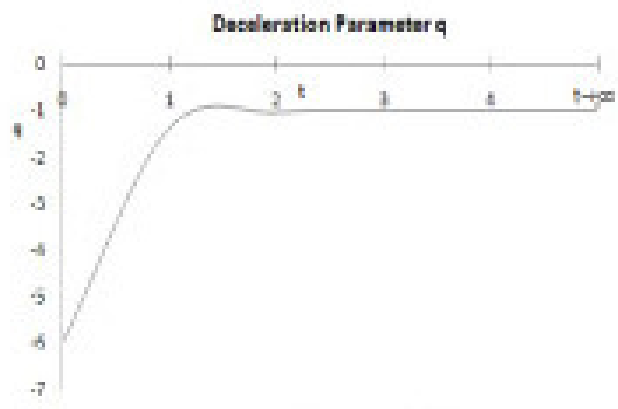

Figure 5. $q$ vst

The graph of scalar expansion $\theta$ s hyperbolic tangential. At $t=0, \theta=0$ and it is increasing hyperbolic tangentially and attains the finite value when $t \rightarrow \infty$. This shows that the mode 
It is well known that the universe underwent an accelerating expansion right after the bigbang (Inflation Era) and at high red shifts (Dark Energy Era). The universe must decelerate $(q>0)$ when radiation dominates its dynamics. Figure 5 reflects quite unpleasant nature of deceleration parameter $q$ which has more negative value in the beginning which shows that the model starts with highly accelerating phase and acceleration is slowing down continuously. We are putting this argument on the basis of our mathematical results and this argument may be unpleasant since nobody knows the secret of the nature and the wonder of the physics of the universe.

Case (iii)For $\quad y=1, \quad$ Equation (25) has a solution

$$
V=\beta \quad\left(\cosh \left(\sqrt{c_{2}} t\right)+1\right),
$$

where $\not$ s positive constant.

From Equations (15), we write

$$
A=B=C=(\not \cosh \quad t+1))^{13},
$$

The required line element $\left(2 \sqrt{s_{2}}\right.$

$$
d s^{2}=-d t^{2}+\left(\beta\left(\cosh \quad \sqrt{c_{2}} t+1\right)\right)^{23}\left(d x^{2}+d y^{2}+d z^{2}\right)
$$

This is the Bianchi type I cosmological model with perfect fluid (stiff matter) in biometric theory of gravitation. This Zeldovich universe has volumetric hyperbolic expansion (as shown in Figure 6 similar to that of the radiating universe case (ii). The scale factors also have similar behavior as case (ii). The model starts with nonzero volume and volume increases in hyperbolic cosine nature and has infinite values at the finalstage.

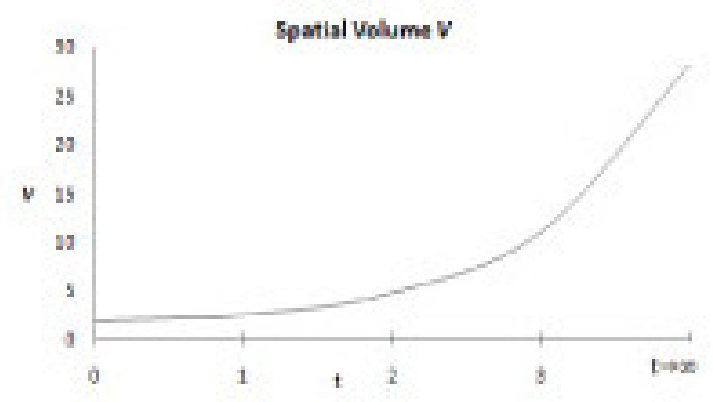

Figure 6, $V$ vs $t$

The energy density $\rho_{\rho F}$ and the pressure $p_{\rho F}$ of the perfect fluid (stiff matter) are

$$
\rho_{\text {FF }}=p_{F F}=c_{1} / \beta^{2}\left(\cosh \left(\sqrt{c_{2}} t\right)+1\right)^{2}
$$

The physical parameters are 


$$
\begin{aligned}
& \theta=\frac{6 \operatorname{cosinh}\left(c_{\sqrt{3}}\right)}{(\cosh (\sqrt{2} t)+1)}, \\
& A=\sigma=0, \\
& q=-\frac{3}{\cosh \left(\sqrt{c_{2}} t\right)-1}-1 .
\end{aligned}
$$

In this perfect fluid Zel'dovich universe, it is observed that all the physical parameters $\rho_{P F}, p_{P F}, \theta$ and decelerating parameter $q$ behave in similar nature as that of case (ii) of radiating universe and having the nature of hyperbolic functions as explained earlier in case (ii) and there is no new contribution regarding the geometrical and physical behavior of these parameters, whose graphs are shown below.

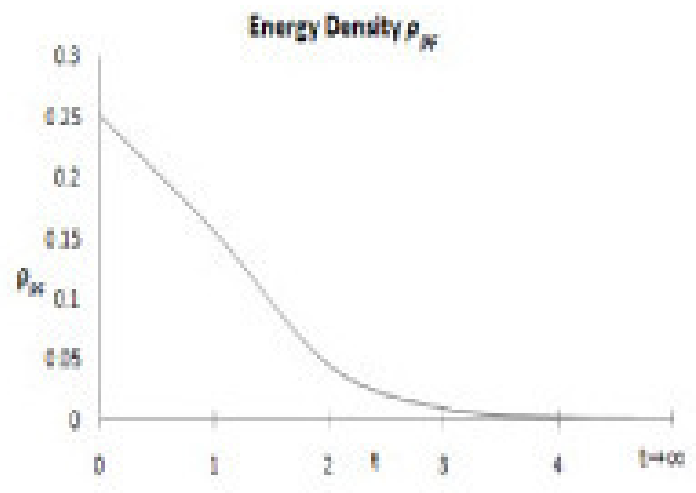

Figure 7, $\rho_{P E} \mathrm{vst}$

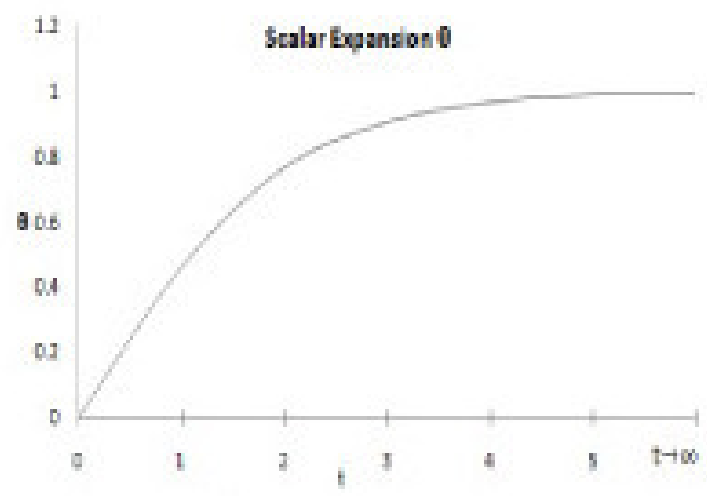

Figure 9. Avst

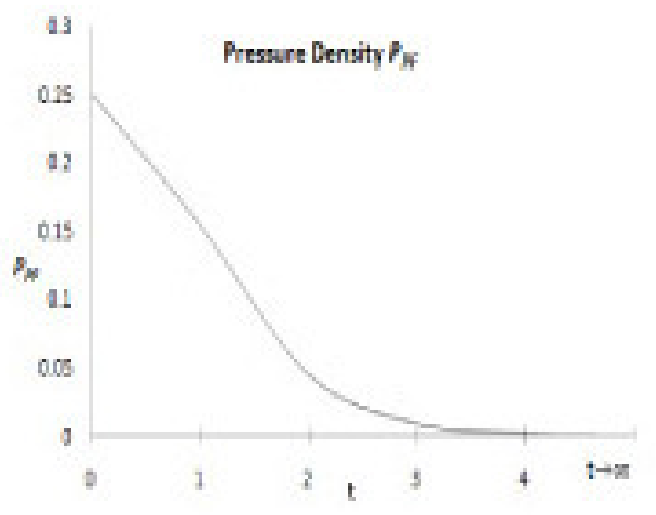

Figure8, $p_{P F} \mathrm{vs} t$

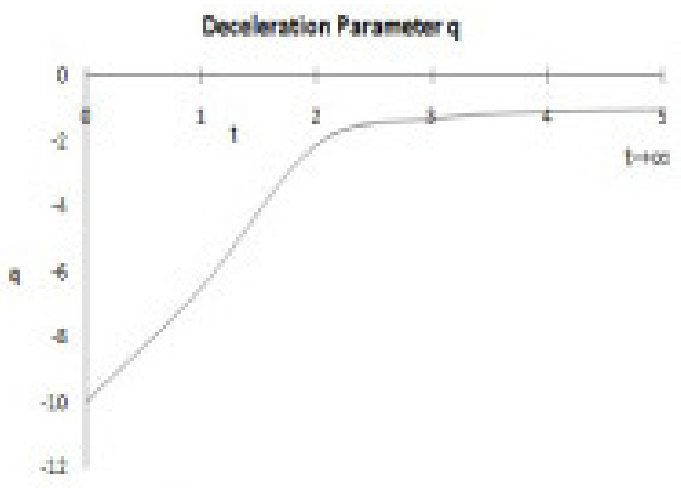

Figure 10. $q$ vst

It is important to say that in these perfect fluid models (29) and (36) corresponding to radiating universe and Zel'dovich universe, the geometry and all the physical parameters of the models behave hyperbolically in nature, since hyperbolic geometric functions are present in it. Therefore, the geometrical and physical properties of the models with physical 
parameters have been studied from hyperbolic geometric view point. This is the remarkable point observed in the geometry of the model that the model has hyperbolic geometry and it is helpful to the people of observational data to search such type of universe.

\section{Summary}

1. Theperfectfluidmodelscorrespondingto $y=1 / 3$ (radiating)and $y=1$ (stiffmatter)are hyperbolicgeometricinnature. Theperfectfluidmodelcorrespondenceto $y=0$ (dust) does notexist.

2. All the physical matters in these perfect fluid models obey the graph of hyperbolic geometric functions and therefore their natures have been studied from hyperbolic geometric viewpoint.

3. These perfect fluid models have volumetric hyperbolicexpansion.

4. These perfect fluid models are isotropize in nature withoutshear.

5. These perfect fluid models are highly accelerating and acceleration of the model goes on increasing as time $t$ is increasing and has the constant acceleration at later stages of timet.

\section{Conclusion}

Bianchi type-I cosmological models corresponding to perfect fluid in Bimetric theory of gravitation have been deduced. The perfect fluid model has hyperbolic geometry and all its physical parameters are also hyperbolic in nature and therefore they have been studied from a hyperbolic geometric view point.

All these models are isotropize and shear-less. Other geometrical and physical behaviors of the models have been studied.

\section{REFERENCES}

Ade P. A. R., Aghanim, N., Armitage-Caplan, C., Arnaud M., (2014). Planck 2013results.

XVI. Cosmological parameters, Astronomy and Astrophysics, Vol, 571, pp. Al6 Adelman-McCarthy, J. K. Agüeros, M. A., Allam, S. S., Anderson, K. S. J., Anderson, S. F.,

Annis, J., Bahcall, N. A., Baldry, I. K.,Barentine, J. C., Berlind, A., Bernardi, M., Blanton, M. R.,Boroski, W. N., Brewington, H. J., Brinchmann, J., Brinkmann, J., Brunner, R.J., Budavári, T.,Carey, L.N.,Carr, M. A., Castander, F.J., Connolly, A. J., Csabai, I.,Czarapata, P.C., Dalcanton, J.J., Doi, M., Dong, F., Eisenstein, D.J., Evans, M.L.,Fan, X.Finkbeiner, D.P.,Friedman, S.D., Frieman, J.A., Fukugita, M., Gillespie, B.,Glazebrook, K., Gray, J., Grebel, E.K.,Gunn, J.E., Gurbani, V.K., de Haas, E., Hall, P. B., Harvanek, M.,Hawley, S.L.,Hayes, J., Hendry, John S., Hennessy, G.S.,Hindsley, R.B.,Hirata, C.M., Hogan, C. J., Hogg, D.W., Holmgren, D. J., Holtzman, J.A., Ichikawa, S., Ivezič, Ż.,Jester, S., Johnston, D. E., Jorgensen, A.M.,Jurić, M., Kent, S.M., Kleinman, S. J., Knapp, G. R.,Kniazev, A. Y.,

Kron, R.G.,Krzesinski, J., Kuropatkin, N., Lamb, D.Q., Lampeitl, H., Lee, B.C., Leger, R. F.,Lin, H.,Long. D.C.,Loveday, J., Lupton, R.H.,Margon, B., Vanden, B., 
Akarsu, O. and Kilinc, C. B. (2010). LRS bianchi type I models with anisotropic dark energy and constant deceleration parameter, General Relativity and Gravitation, Vol. 42, No.1, pp. 119.

Akarsu, O. and Kilinc, C. B. (2010). Bianchi type III models with anisotropic dark energy, General Relativity and Gravitation, Vol. 42, No. 4, pp. 763.

Akarsu, O. and Kilinc, C.B. (2010). De-sitter expansion with anisotropic fluid in bianchi type I space time, Astrophysics and Space Science, Vol. 326, No. 2, pp. 315.

Bali, R. and Dave, S. (2003). Bianchi type-IX string cosmological models with bulk viscous fluid in general relativity, Astrophysics and Space Science, Vol. 288, pp. 503.

Bali, R and Upadhaya, R.D. (2003). LRS bianchi type I string dust magnetized cosmological models, Astrophysics and Space Science, Vol. 283, pp. 97.

Bali, R. and Singh, D. (2005). Bianchi type-V bulk viscous fluid string dust cosmological model in general relativity, Astrophysics and Space Science, Vol. 300, pp. 387.

Bali, R and Anjali (2006). Bianchi type I magnetized string cosmological model in general relativity, Astrophysics and Space Science, Vol. 302, pp. 201.

Bali, R. and Pareek, U. K. (2007). Bianchi type I string dust cosmological model with magnetic field in general relativity, Astrophysics and Space Science, Vol. 312, pp. 305.

Bennett, C. L., Hill R. S., Hinshaw G., Nolta M. R., Odegard N., Page L., Spergel D. N., Weiland J. L., Wright E. L., Halpern M., Jarosik N., Kogut A., Limon M., Meyer S. S., Tucker G. S. and Wollack E., (2003). First year Wilkinson anisotropy probe observations: Foreground Emission, The Astrophysical Journal Supplement Series, Vol. $148, \mathrm{pp} .97$.

Borkar, M. S. and Charjan, S. S. (2010). Bianchi type I magnetized cosmological model in bimetric theory of gravitation, Application and Applied Mathematics (AAM), Vol. 5, No. 2 , pp. 563.

Borkar, M. S. and Charjan, S. S. (2010). Bianchi type I bulk viscous fluid string dust cosmological model with magnetic field in bimetric theory of gravitation, Applications and Applied Mathematics (AAM), Vol. 5, No. 9, pp. 96.

Gaikwad, N. P. and Borkar, M. S. (2011). Bianchi type I massive string magnetized baratropic perfect fluid cosmological model in bimetric theory of gravitation, Chinese Physics Letter, Vol. 28, N. 8, pp.089803.

Borkar, M. S., Charjan, S. S. and Lepse, V. V. (2013). Evaluation of bianchi type $\mathrm{VI}_{0}$ cosmological models with a binary mixture of perfect fluid and dark energy in bimetric theory of gravitation, International Journal of Applied Mathematics, Vol. 28, No. 2, pp. 2051.

Borkar, M. S. and Gayakwad, P. V. (2014). Evaluation of Schwarz child's exterior and interior solutions in bimetric theory of gravitation, Applications and Applied Mathematics (AAM), Vol. 9, No. 1, pp.260. 